\title{
Allogenic Stem Cell Transplantation and the Subsequent Paraproteinemia: A Benign Altered B-Cell Homeostasis?
}

\author{
Corinne C. Widmer \\ Clinic of Medical Oncology and Hematology, Division of Hematology, University Hospital Zurich and University of \\ Zurich, Zurich, Switzerland
}

For a very long time, the de novo development of paraproteinemia has been observed after allogenic stem cell transplantation (allo-HSCT) of non-myeloma patients and even in patients with solid organ transplantations [1,2]. Its appearance is likely linked to the profound immunodeficiency that these patients experience, but the causal trigger and clinical impact remain poorly understood. Earlier studies described the association of paraproteinemia in patients with allo-HSCT with an alemtuzumab-based conditioning regimen and cytomegalovirus (CMV) reactivation [3]. However, even after the emergence of other conditioning regimens and the following reduction of the use of alemtuzumab over the past decades, paraproteinemia remained a continuously observed phenomenon after allo-HSCT. Newer studies suggest that the process of immunological reconstitution of the individual patient itself with an altered B-cell homeostasis might lead to a selected production of immunoglobulins $[4,5]$.

The fact that it can be easily monitored in the peripheral blood makes paraproteinemia a very promising target for research and the clinical and prognostic impact of this biomarker needs further investigation. The report by Iovino et al. [6] in this issue of Acta Haematolgica is a rep-

\section{KARGER}

(c) 2018 S. Karger AG, Basel

E-Mail karger@karger.com

www.karger.com/aha lication study probing this phenomenon. Their results are in line with another study recently published by our group [5] and confirmed a prognostic relevance. The statistical analysis of 194 patients revealed a better outcome in patients with paraproteinemia in the first year after allo-HSCT and this trend was also visible for the 5-year overall survival. Furthermore, there was no evidence of an association of paraproteinemia with the conditioning regimen or with infectious conditions such as CMV reactivation. Other clinical parameters such as graft versus host disease or underlying diseases were also not significantly connected; however, the small size of subgroups needs to be considered. It remains to be investigated though which immune system reconstitutions will respond with a paraprotein production and therefore provide an improved outcome in the first year after alloHSCT. The overall incidence of paraproteinemia in the study by Iovino et al. [6] was low and, accordingly, the statistical power was not high enough to make a statement about predicting factors for a paraproteinemia. The authors themselves indicated that due to the already observed transient nature of the paraproteinemia, they might have missed short-lasting appearances of paraproteins. A profound immunodeficiency is expected to be 
prevalent for at least 3 months after transplantation and it would be interesting to investigate the development of paraproteinemia within these first 3 months. Accordingly, I would encourage screening for paraproteinemia from the beginning, possibly with shorter intervals to improve understanding of its kinetics and development. The fact that the appearance of paraproteinemia mostly occurs within the first year after allo-HSCT might hint to the kinetics of B-cell reconstitution.

Even though there are more and more patients eligible for allogenic transplantation, there are very limited data on this topic. While paraproteinemia after allo-HSCT is mostly transient and does not translate into the development of plasma cell myeloma or lymphoma, further understanding of its cause and impact on the disease course of the patient is of high prognostic interest. The study by Iovino et al. [6] provides another piece to the puzzle and helps to consolidate the benign character of this paraproteinemia. Even though their sample size was rather low, the importance of such studies should not be underestimated as they lay the foundation for subsequent metaanalyses that might then be able to make statements of high clinical and statistical significance.

Furthermore, it raises interest to gain more insight into this particular immune reconstitution, even perhaps leading to an overall better understanding of the development of monoclonal gammopathies in general and, additionally, might yield an easily assessable prognostic factor for patients after allo-HSCT.

\section{Disclosure Statement}

The authors have no conflicts of interest to declare.

\section{References}

1 Pageaux GP, Bonnardet A, Picot MC, Perrigault PF, Coste V, Navarro F, et al. Prevalence of monoclonal immunoglobulins after liver transplantation: relationship with posttransplant lymphoproliferative disorders. Transplantation. 1998 Feb;65(3):397-400.

2 Gerritsen EJ, van Tol MJ, Lankester AC, van der Weijden-Ragas CP, Jol-van der Zijde CM, Oudeman-Gruber NJ, et al. Immunoglobulin levels and monoclonal gammopathies in children after bone marrow transplantation. Blood. 1993 Dec;82(11):3493-502.
3 Medd P, Littlewood S, Danby R, Malladi R, Clifford R, Wareham D, et al. Paraproteinaemia after allo-SCT, association with alemtuzumab-based conditioning and CMV reactivation. Bone Marrow Transplant. $2011 \mathrm{Jul}$; 46(7):993-9.

4 Sarantopoulos S, Stevenson KE, Kim HT, Cutler CS, Bhuiya NS, Schowalter M, et al. Altered B-cell homeostasis and excess BAFF in human chronic graft-versus-host disease. Blood. 2009 Apr;113(16):3865-74.
5 Widmer CC, Balabanov S, Schanz U, Theocharides AP. Transient paraproteinemia after allogeneic hematopoietic stem cell transplantation is an underexplored phenomenon associated with graft versus host disease. Oncotarget. 2017 Nov;8(63):106333-41.

6 Iovino L, Mazziotta F, Buda G, Orciuolo E, Caracciolo F, Pelosini M, et al. The onset of monoclonal and oligoclonal gammopathies after allogeneic stem cell transplantation represents a good prognostic factor. Acta Hematol. 2018. doi: 10.1159/000493416. 